\title{
DIAGNOSTIC VALUE OF NEUTROPHIL TO LYMPHOCYTE RATIO TO RULE OUT CHRONIC OBSTRUCTIVE PULMONARY DISEASE EXACERBATION FROM ACUTE HEART FAILURE IN THE EMERGENCY DEPARTMENT
}

\author{
Erdal Demirtaş ${ }^{1}\left(\mathbb{D}\right.$, Esin Demirtaş $^{2}$ (1) \\ ${ }^{1}$ Department of Emergency Medicine, Sivas Cumhuriyet University Faculty of Medicine, Sivas, Turkey \\ ${ }^{2}$ Department of Family Medicine, Sivas Cumhuriyet University Faculty of Medicine, Sivas, Turkey
}

\begin{abstract}
INTRODUCTION: This study was performed to determine whether neutrophil-lymphocyte ratio (NLR) optimizes the differential diagnosis of acute chronic obstructive pulmonary disease (COPD) exacerbation and acute heart failure (AHF) in patients admitting to the emergency department (ED) with dyspnoea.

MATERIAL AND METHODS: The study group included 241 patients (135 males and 106 females) who were admitted to the ED with a complaint of dyspnoea between January 2016 November 2018 and were hospitalized with diagnosis of acute COPD exacerbation or AHF.

RESULTS: White blood cell count, neutrophil, haemoglobin, haematocrit, NLR, and C-reactive protein values in patients with COPD exacerbation were significantly higher compared to those with AHF $(p<0.05)$. The largest areas under the ROC curve were obtained with NLR. The cut-off value for NLR was 9.39, with a sensitivity of $71 \%$ and a specificity of $61 \%$.

CONCLUSIONS: In conclusion, the NLR has diagnostic value to the conventional clinical assessment in patients with acute dyspnoea at ED, especially with acute COPD exacerbation requiring inpatient management. This may be an additional tool during the differential diagnosis of dyspnoea for emergency physicians in hospitals without advanced laboratory facilities.
\end{abstract}

KEY WORDS: neutrophil-lymphocyte ratio; dyspnoea; acute COPD exacerbation; acute heart failure; emergency medicine

Disaster Emerg Med J 2019; 4(3): 102-108

\section{INTRODUCTION}

The diagnosis and management of patients admitting to the emergency departments (ED) with acute dyspnoea and accompanying hypoxemia are complicated due to the wide spectrum of differential diagnosis. Following stabilization of the airway, breathing, and circulation in a dyspnoeic patient, further etiological investigation including acute heart failure
(AHF), pneumonia, and exacerbation of chronic obstructive pulmonary diseases (COPD) can proceed. Differential diagnosis should be prompt while providing appropriate first-line treatment for possibly lethal conditions [1].

Early ruling out of patients with COPD exacerbation from AHF for emergent medical intervention can be essential for emergency physicians in the 
ED. As limited diagnostic information is available to emergency physicians for making decisions, there is a need for reliable diagnostic markers in patients with symptoms, mainly dyspnoea, suggestive of a cardiopulmonary pathology. There are several studies emphasizing the importance of haematological biomarkers for differential diagnosis in patients with dyspnoea [2-4].

The neutrophil to lymphocyte ratio (NLR) is calculated by dividing the number of neutrophils (NEU) by a number of lymphocytes (LYM) in a peripheral blood sample. The NLR is a simple parameter to assess the inflammatory status of a subject easily because it is relatively inexpensive and widely available as a part of a routine blood count analysis [5]. Furthermore, NLR has been demonstrated to be superior to routine parameters like C-reactive protein (CRP) level, white blood cell (WBC) count and NEU count in predicting bacteraemia [6].

It is not always possible to immediately evaluate every dyspnoeic patient with costly and complex clinical assessments (echocardiography, nuclear scans, or cardiac catheterization) in the ED; besides, the prognostic benefit of these remains uncertain. Therefore, the authors sought to measure the additive prognostic value provided by haematological parameters including NLR and PLR in patients with dyspnoea. The objective of this study determined whether NLR optimizes the differential diagnosis of acute chronic obstructive pulmonary disease (COPD) exacerbation and acute heart failure (AHF) utilizing clinical examination and laboratory parameters as main diagnostic tools in dyspnoeic patients admitting to the ED of a university hospital.

\section{MATERIALS AND METHODS}

This was a retrospective cross-sectional study performed on patients admitted to ED of a university hospital with complaints of dyspnoea due to COPD exacerbation or systolic AHF between January 2016 November 2018. After an initial evaluation, all patients were managed either in Department of Pulmonary Disease or Department of Cardiology. Patients with pulmonary embolism, acute myocardial infarction, AHF unstable angina, history of AHF and COPD, pneumonia, AHF and infection, history of renal failure, lung cancer, more than 5 packet/ year smoker history, pregnancy, asthma and rhinitis, or those with missing complete blood count (CBC) values, were excluded from the study (Fig. 1).

Acute exacerbation of COPD was defined as an event in the natural course of the disease characterized by a change in the patient's baseline respiratory symptoms such as dyspnoea, cough, sputum production, volume and colour change, that may warrant a change in regular medication in a patient with underlying COPD [7]. Acute heart failure is defined more broadly as a new onset or gradual or rapidly worsening HF [8].

\section{5 patients additted to emergency service because of dyspnea in 2016-2018}
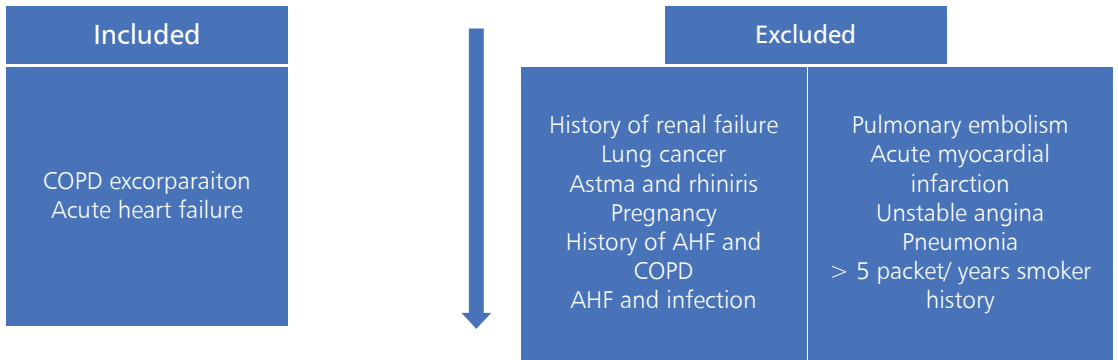

Patients presented with dyspnea and admitted to hospital with a diagnosis of COPD exacerbation or acute heart failure $(n=241)$

COPD exacerbation $(n=139)$

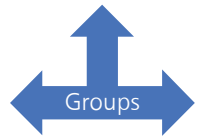

Acute heart failure $(n=102)$

FIGURE 1. Schematic presentation of patient enrolment to the acute chronic obstructive pulmonary disease exacerbation and acute heart failure groups 
Following approval from the local ethics committee (2018-12/10), data were collected retrospectively from the hospital database. The recorded data were as follows: haemoglobin, leukocytes, neutrophils, lymphocytes, haemoglobin, mean platelet volume, and platelet distribution width (Mindray BC6800, China). NLR was calculated by dividing the absolute neutrophil count by the absolute lymphocyte count. PLR was calculated by dividing the absolute platelet count by the absolute lymphocyte count. CRP level was calculated with the nephelometry technique (Beckman Coulter, California, USA). Albumin, alanine aminotransferase (ALT), aspartate aminotransferase (AST), $\gamma$-glutamyl transferase (GGT), glucose, direct bilirubin, total bilirubin, blood urea nitrogen (BUN), serum creatinine, sodium, potassium, creatine kinase (CK), CK-MB, troponin levels were determined with the enzymatic colorimetric method (Mindray BS2000, USA). Blood gas analysis was performed (Radiometer ABL850 USA). Same methodology and devices were used for all the laboratory parameters during the search period.

\section{STATISTICAL ANALYSIS}

IBM SPSS Statistics for Windows version 23.0 (IBM, Armonk, NY, USA) was used for statistical analysis. Kolmogorov-Smirnov test was used as a test for normality for continuous data, and the results were expressed as median with 25th and 75th percentiles. Non-parametric Mann-Whitney $U$ test was used to compare the means between two groups. Receiver operating characteristic (ROC) analysis was used to determine the diagnostic efficiency of NLR, CRP, WBC, and PLR, and the areas under the curve (AUCS) were compared. $P<0.05$ was considered statistically significant.

\section{RESULTS}

There was no significant difference between the patients with acute COPD exacerbation and AHF regarding mean age $(70.5+13.7$ and $71.7+11.3$ years respectively) and female to male ratio $(0.43$ and 0.56 , respectively) $(p>0.05)$. Table 1 and Figure 2 present $\mathrm{CBC}$ and biochemistry test results. The white blood cell (WBC), neutrophil, haematocrit, and NLR values in patients with acute COPD exacerbation were significantly higher than in the patients with AHF $(p<0.05)$. The eosinophil and MCV value in patients with acute COPD exacerbation were significantly lower than that in patients with AHF $(p<0.05)$. The haemoglobin, lymphocyte, monocyte, basophil, haemoglobin, PLR, and platelet values of the study groups were comparable $(p>0.05)$. GGT, direct bilirubin, total bilirubin, serum creatinine, $\mathrm{CK}$, and troponin levels in patients with COPD exacerbation were significantly lower than in patients with AHF $(p<0.05)$. The CRP level in patients with COPD exacerbation was significantly higher than in patients with AHF $(p<0.05)$. AST, ALT, glucose, albumin, sodium, potassium, and CK-MB levels of the study groups were comparable $(p>0.05)$. Table 2 presents the blood gas parameters in acute COPD exacerbation and AHF. $\mathrm{pCO}_{2}, \mathrm{COHb}$, and $\mathrm{HCO}_{3}$ values in patients with COPD exacerbation were significantly higher than in patients with AHF $(p<0.05)$. The $p H$, $\mathrm{O}_{2} \mathrm{Hb}, \mathrm{sO}_{2}$ and $\mathrm{pO}_{2}$ values of the study groups were comparable $(p>0.05)$.

In addition, receiver operating characteristic curve (ROC) analyses with area under the curve (AUC) comparisons were used for determining the optimum cut-off value for CRP, NLR, WBC in predicting acute COPD exacerbation in patients with dyspnoea (Fig. 3). Among these inflammatory parameters, NLR had significance with the largest AUCs $(p<0.05)$. The cut-off value for NLR was 9.39 , with a sensitivity of $71.7 \%$ and a specificity of $61.1 \%$ and areas under the ROC curve: 0.716 . The cut-off value for CRP was 25.7 , with a sensitivity of $58.6 \%$ and a specificity of $57.6 \%$, and areas under the ROC curve: 0.593 . ROC analysis for WBC is comparable ( $p>0.05)$.

\section{DISCUSSION}

In this study including 139 patients with acute COPD exacerbation and 102 patients with AHF hospitalized for management after the initial evaluation, the authors investigated the value of NLR as a diagnostic parameter for differential diagnosis in the ED. The age and sex ratio of study groups were comparable, and the inflammatory parameters including neutrophil values and CRP level increased in patients with acute COPD exacerbation. These findings enhance the clinical importance of higher NLR in patients with acute COPD exacerbation as a diagnostic parameter to differentiate these patients from patients with AHF. Other clinical parameters were supportive of the clinical nature of acute COPD exacerbation and AHF diseases. NLR can predict the patients with acute COPD exacerbation with a sensitivity and specificity of $71 \%$ and $61 \%$, respectively. CRP level sen- 
Table 1. Baseline characteristics of COPD exacerbation and acute heart failure

\begin{tabular}{|c|c|c|c|}
\hline & $\begin{array}{l}\text { COPD exacerbation } \\
\qquad(\mathrm{N}=139)\end{array}$ & $\begin{array}{l}\text { Acute Heart Failure } \\
\qquad(\mathrm{N}=102)\end{array}$ & $p$ \\
\hline $\begin{array}{l}\text { CBC, median (IQR) values } \\
\text { WBC } \\
\text { Neutrophil count } \\
\text { Lymphocyte count } \\
\text { Monocyte count } \\
\text { Eosinophil count } \\
\text { Basophilic count } \\
\text { Haemoglobin, g/dL } \\
\text { Haematocrit, \% } \\
\text { MCV, fL } \\
\text { Platelet count, } 10^{9} \\
\text { NLR } \\
\text { PLR }\end{array}$ & $\begin{array}{l}11.2(8.2-14.4) \\
9.5(6.7-13.3) \\
1(0.57-1.37) \\
0.49(0.25-0.76) \\
0.02(0.01-0.08) \\
0.03(0.02-0.05) \\
13.8(12.2-15.5) \\
43.9(39-50.3) \\
87.1(80.3-91.5) \\
229(170-292) \\
12.6(8.4-22.9) \\
230(146-392)\end{array}$ & $\begin{array}{l}9.8(7.9-12.4) \\
7.7(5.8-10.7) \\
1.02(0.67-1.74) \\
0.56(0.41-0.74) \\
0.07(0.02-0.12) \\
0.03(0.02-0.05) \\
12.8(11.5-14.9) \\
41.2(36.3-47.3) \\
88.9(83.6-92.7) \\
228(181-290) \\
8.1(4.5-13.6) \\
224(126-342)\end{array}$ & $\begin{array}{l}0.068 \\
0.003 \\
0.276 \\
0.107 \\
0.041 \\
0.806 \\
0.054 \\
0.008 \\
0.037 \\
0.418 \\
<0.001 \\
0.065\end{array}$ \\
\hline $\begin{array}{l}\text { Biochemistry } \\
\text { Glucose, mg/dL } \\
\text { Albumin, g/dL } \\
\text { ALT, U/L } \\
\text { AST, U/L } \\
\text { GGT, U/L } \\
\text { Direct bilirubin, mg/dL } \\
\text { Total bilirubin, mg/dL } \\
\text { BUN, mg/dL } \\
\text { Serum creatinine, mg/dL } \\
\text { Sodium, mmol/L } \\
\text { Potassium, mmol/L } \\
\text { CK } \\
\text { CKMB } \\
\text { Troponin } \\
\text { CRP, mg/dL }\end{array}$ & $\begin{array}{l}132(115-156) \\
4.05(3.8-4.2) \\
17(9-27) \\
23(18-32) \\
34(22-61) \\
0.23(0.15-0.43) \\
0.63(0.40-0.99) \\
23.6(17.1-34.3) \\
0.95(0.73-1.28) \\
136(133-139) \\
4.3(3.9-4.7) \\
58(41-99) \\
32(21-42) \\
0.03(0.02-0.05) \\
42.4(13.1-88.6)\end{array}$ & $\begin{array}{l}131(102-381) \\
3.81(3.17-4.04) \\
18(12-36) \\
28(21-44) \\
56(27-105) \\
0.28(0.19-0.54) \\
0.78(0.50-1.09) \\
28.2(19.8-40.2) \\
1.13(0.87-1.63) \\
135(133-139) \\
4.2(3.8-4.6) \\
77(49-171) \\
32(24-44) \\
0.05(0.03-0.09) \\
22.1(14.5-44.8)\end{array}$ & $\begin{array}{l}0.767 \\
0.370 \\
0.075 \\
0.193 \\
<0.001 \\
0.003 \\
0.006 \\
0.013 \\
0.031 \\
0.95 \\
0.241 \\
0.005 \\
0.636 \\
0.021 \\
<0.001\end{array}$ \\
\hline
\end{tabular}

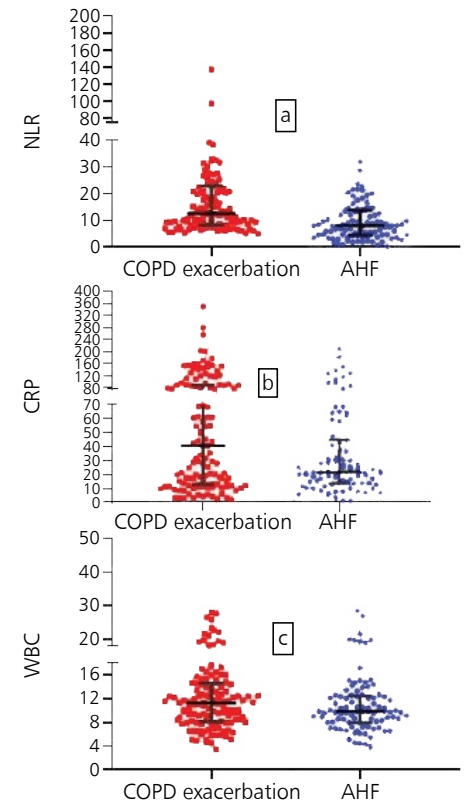

FIGURE 2. Neutrophil to lymphocyte ratio (NLR), white blood cell (WBC), and C-reactive protein (CRP) inflammatory markers in chronic obstructive pulmonary disease (COPD) exacerbation acute heart failure (AHF). a,b, $P<0.05, c P>0.05$ vs AHF sitivity and specificity (58\% and 57\%, respectively). Overall, NLR as an inflammatory parameter, especially in emergency room settings with basic laboratory conditions, has merit to be used for easy and rapid differential diagnosis between patients with acute COPD exacerbation and AHF. Besides, its clinical implementation can decrease prolonged stays in the ED, reduce unnecessary hospitalizations, inappropriate discharges, thus leading to better patient care.

Dyspnoea is a common chief complaint as an uncomfortable subjective breathing sensation that is distressful or unpleasant in nature among ED patients [9]. Emergency clinicians need to establish the most likely causes of dyspnoea and initiate treatment in such a patient. In clinical practice, COPD is a frequent disease characterized by persistent respiratory symptoms including dyspnoea [10]. Patients with COPD are easily affected by many conditions that can lead to acute deterioration of the underlying COPD. Although COPD exacerbations are very 


\begin{tabular}{|c|c|c|c|}
\hline & COPD exacerbation $(\mathrm{N}=165)$ & $\begin{array}{l}\text { Acute Heart Failure } \\
\qquad(\mathrm{N}=115)\end{array}$ & $P$ Value \\
\hline $\begin{array}{l}\mathrm{pH} \\
\mathrm{pCO}_{2} \\
\mathrm{pO}_{2} \\
\mathrm{COHb} \\
\mathrm{HCO}_{3} \\
\mathrm{O}_{2} \mathrm{Hb} \\
\mathrm{SO}_{2}\end{array}$ & $\begin{array}{l}7.42(7.36-7.45) \\
45(34-58) \\
54.1(44.7-67) \\
2.2(1.8-2.8) \\
26(22-31) \\
82(74-88) \\
84(75-90)\end{array}$ & $\begin{array}{l}7.41(7.36-7.46) \\
35.1(31.3-45.1) \\
59.9(51.3-70.1) \\
2(1.7-2.5) \\
22.5(19.2-25.3) \\
86(79-90) \\
87(81-93)\end{array}$ & $\begin{array}{l}0.407 \\
<0.001 \\
0.673 \\
0.001 \\
<0.001 \\
0.103 \\
0.138\end{array}$ \\
\hline
\end{tabular}

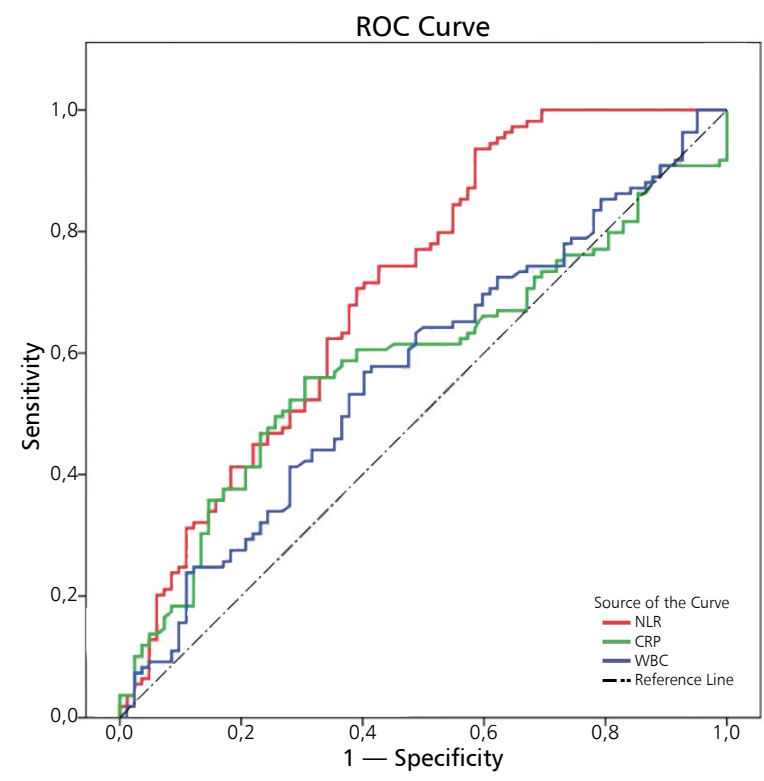

FIGURE 3. ROC curves of inflammatory markers for acute COPD exacerbation patients to AHF. Abbreviations: ROC, receiver operating characteristic; CRP, C-reactive protein; WBC, white blood cell NLR; neutrophil to lymphocyte ratio. ROC analysis for NLR $(P<0.001$, areas under the ROC curve: 0.716 ; sensitivity: $71.7 \%$ and specificity: $61 \%$ for a cut-off of 9.39 ). ROC analysis for CRP $(P=0.021$, areas under the ROC curve: 0.593 ; sensitivity: $58.6 \%$ and specificity: $57.6 \%$ for a cut-off of 25.7$)$. ROC analysis for WBC $(P=0.092)$

common (about $20 \%$ of patients) and important, they are usually overlooked. Rapid and proper recognition of these patients along with appropriate treatment may be the only action that prevents respiratory failure and mortality [11-13]. Currently, there is no consensus for the assessment of the severity and progression of acute COPD exacerbations by using a clinical tool [5].

Heart failure is also a critical clinical condition with findings such as dyspnoea, orthopnoea, lower limb swelling, and pulmonary congestion because of possible structural and/or functional cardiac abnor- mality with important physiological abnormalities including reduced cardiac output and/or elevated intracardiac pressures [14]. Dyspnoea is the essential manifestation of $\mathrm{HF}$ and remains its most perplexing feature.

COPD and HF are global epidemics, each affecting more than 10 million patients [15]. In addition, both diseases often have common symptoms, and there is often significant overlap in the findings [16]. In order to prevent delays in treatment, objective and adequate diagnostic methods are needed in patients with unusual clinical manifestations and coinciding pathologies. Clinical, laboratory and radiographic findings that contradict the clinician's initial impressions must be carefully considered, as misdiagnosis may be associated with increased morbidity and mortality.

Kelly et al. [17] assessed the demographics and clinical presentation, assessment, treatment, and outcome of patients with acute COPD exacerbation managed in the EDs of Australasia and South East Asia. They included forty-six EDs to their research and found 415 eligible patients (13.6\% of the overall cohort) with a primary diagnosis of acute COPD exacerbation ( $91 \%$ of them with previous COPD diagnosis) in the ED. The authors noted that those patients commonly required inpatient management and their in-hospital mortality was pronounced. They highlighted the suboptimal compliance of those patients to their suggested management protocols.

Laribi et al. [18] evaluated the epidemiology and management of dyspnoeic patients presenting to EDs in an international patient population in Europe and the Asia-Pacific region. Among 5569 patients, the most common ED diagnoses were lower respiratory tract infection (24.9\%), heart failure (17.3\%), COPD exacerbation (15.8\%), and asthma (10.5\%). 
They found that the overall in-hospital mortality was $5.0 \%$, especially $7.6 \%$ for heart failure and $5.6 \%$ for COPD patients. They suggested that exacerbations of chronic diseases including HF and COPD account for a large proportion of dyspnoeic patients in their population. They noted that dyspnoea at the ED was associated with a high rate of ward admission as well as suboptimal short-term outcomes. Similarly, all the patients in this study population were admitted for inpatient management.

Patients with a COPD exacerbation may present with dyspnoea and signs of right ventricular volume overload due to worsening cor pulmonale. In the management of these patients, laboratory testing should be performed in the context of the history and examination findings. Testing without a clear differential diagnosis can mislead the clinician and delay appropriate management. The use of dyspnoea biomarker panels does not appear to improve accuracy beyond clinical assessment and focused testing [19]. Nevertheless, a plain chest radiograph and an electrocardiogram are obtained in most ED patients with dyspnoea. Biomarkers are objective indications of the medical state of the patient. Natriuretic peptides, brain natriuretic peptide (BNP) and N-terminal prohormone of brain natriuretic peptide (NT-proBNP), have been accepted as the gold standard in assessing patients with heart failure. However, different cut-off values accepted for acute and chronic manifestations of heart failure are the main limitation of these biomarkers. Emergency evaluation of patients with AHF in the ED may be suboptimal because of high-flow of patients, prolonged waiting time, and a deficit of medical staff [20]. In these patients, BNP levels are likely to be high, though usually not as high as cardiac dyspnoea [21]. The authors believe that simple and inexpensive laboratory tests like the NLR have potential for widespread use in every level of EDs since other biomarkers still require further research for their optimization in patients with dyspnoea related to different types of aetiologies.

NLR has been used in patients with acute COPD exacerbation in several studies, mostly as an indicator of exacerbation. Teng et al. [5] examined the predictive value of NLR in patients with acute COPD exacerbation. Their findings showed that NLR was higher in patients during admission and decreased after treatment according to clinical improvement. They suggested that NLR could be obtained quickly without any important burden to the patients and emergency clinicians, as it is convenient and relatively inexpensive blood routine test. They concluded that NLR could also improve diagnostic accuracy when evaluating the severity and prognosis of acute COPD exacerbation with strong support for formulating a treatment protocol in an emergency setting. They highlighted the importance of potential use of NLR as an easy test in community hospitals. Taylan et al. [22] assessed the place of NLR in patients with COPD for identifying recognition of acute exacerbations. They found that NLR and other inflammatory markers, such as WBC, CRP, and ESR were found to be higher in COPD exacerbation compared to stable COPD. They noted that NLR could be used as a marker similar to the other inflammatory markers including $C R P, W B C$, and ESR in patients with acute COPD exacerbation. The findings of the current study support those data obtained from pertinent literature and the NLR can be considered as a tool for differential diagnosis of acute COPD exacerbation in dyspnoeic patients admitted to ED.

In summary, within the initial laboratory workup, NLR as a simple laboratory parameter can be advantageous for emergency clinicians for the differential diagnosis of acute exacerbation of COPD from AHF in patients presented to the ED with dyspnoea. This may be important for emergency physicians in hospitals without advanced laboratory test opportunities.

Conflict of interest: The authors have no conflict of interest to declare.

Declaration of interest: All authors disclose any financial and personal relationships with other people or organizations that could inappropriately influence their work.

Submission declaration: All authors declare that this paper is not published or under consideration for publishing elsewhere.

Journal policies: All authors reviewed in detail the journal policies.

This study was authorized by the medical authorities of the authors' hospital and patients were informed and signed their consent to their data collection.

\section{REFERENCES}

1. MeiLan King Han MK, Dransfield MT, Martinez JF. Chronic obstructive pulmonary disease: Definition, clinical manifestations, diagnosis, and 
staging. Hollingsworth $\mathrm{H}$ (ed.) UpToDate. Waltham, MA: UpToDate Inc. http://www.uptodate.com (22.12.2018).

2. Stokes NR, Dietz BW, Liang JJ. Cardiopulmonary laboratory biomarkers in the evaluation of acute dyspnea. Open Access Emerg Med. 2016; 8: 35-45, doi: 10.2147/0AEM.S71446, indexed in Pubmed: 27307771.

3. Wettersten N, Maisel AS. Biomarkers for Heart Failure: An Update for Practitioners of Internal Medicine. Am J Med. 2016; 129(6): 560-567, doi: 10.1016/j.amjmed.2016.01.013, indexed in Pubmed: 26844635.

4. Peacock WF, De Marco T, Fonarow GC, et al. ADHERE Investigators. Cardiac troponin and outcome in acute heart failure. N Engl J Med. 2008; 358(20): 2117-2126, doi: 10.1056/NEJMoa0706824, indexed in Pubmed: 18480204.

5. Teng $F_{1}$ Ye $H$, Xue T. Predictive value of neutrophil to lymphocyte ratio in patients with acute exacerbation of chronic obstructive pulmonary disease. PLoS One. 2018; 13(9): e0204377, doi: 10.1371/journal. pone.0204377, indexed in Pubmed: 30265703.

6. de Jager CPC, van Wijk PTL, Mathoera RB, et al. Lymphocytopenia and neutrophil-lymphocyte count ratio predict bacteremia better than conventional infection markers in an emergency care unit. Crit Care. 2010; 14(5): R192, doi: 10.1186/cc9309, indexed in Pubmed: 21034463.

7. Aksoy E, Karakurt Z, Gungor $S$, et al. Neutrophil to lymphocyte ratio is a better indicator of COPD exacerbation severity in neutrophilic endotypes than eosinophilic endotypes. Int J Chron Obstruct Pulmon Dis. 2018; 13: 2721-2730, doi: 10.2147/COPD.S170353, indexed in Pubmed: 30233162.

8. Gheorghiade M, Zannad F, Sopko G, et al. International Working Group on Acute Heart Failure Syndromes. Acute heart failure syndromes: current state and framework for future research. Circulation. 2005; 112(25): 3958-3968, doi: 10.1161/CIRCULATIONAHA.105.590091, indexed in Pubmed: 16365214.

9. Pesola GR, Forde AT, Ahsan H. Screening for Shortness of Breath: Stretching the Screening Paradigm to Tertiary Prevention. Am J Public Health. 2017; 107(3): 386-388, doi: 10.2105/AJPH.2016.303625, indexed in Pubmed: 28177820.

10. Global Initiative for Chronic Obstructive Lung Disease (GOLD): Global Strategy for the Diagnosis, Management and prevention of Chronic Obstructive Pulmonary Disease. (2017 REPORT). http://www. goldcopd.org.

11. Kleinschmidt P. Chronic Obstructive Pulmonary Disease (COPD) and Emphysema in Emergency Medicine. https://emedicine.medscape. com/article/807143-overview\#a3 (28.12.2018).

12. Hurst JR, Vestbo J, Anzueto A, et al. Evaluation of COPD Longitudinally to Identify Predictive Surrogate Endpoints (ECLIPSE) Investigators. Susceptibility to exacerbation in chronic obstructive pulmonary disease. N Engl J Med. 2010; 363(12): 1128-1138, doi: 10.1056/ NEJMoa0909883, indexed in Pubmed: 20843247.
13. Fabbri LM, Beghé $B$, Agusti A. Cardiovascular mechanisms of death in severe COPD exacerbation: time to think and act beyond guidelines. Thorax. 2011; 66(9): 745-747, doi: 10.1136/thoraxjnl-2011-200406, indexed in Pubmed: 21653929.

14. Ponikowski P, Voors AA, Anker SD, et al. ESC Scientific Document Group. 2016 ESC Guidelines for the diagnosis and treatment of acute and chronic heart failure: The Task Force for the diagnosis and treatment of acute and chronic heart failure of the European Society of Cardiology (ESC)Developed with the special contribution of the Heart Failure Association (HFA) of the ESC. Eur Heart J. 2016; 37(27): 2129-2200, doi: 10.1093/eurheartj/ehw128, indexed in Pubmed: 27206819.

15. Hawkins NM, Petrie MC, Jhund PS, et al. Heart failure and chronic obstructive pulmonary disease: diagnostic pitfalls and epidemiology. Eur J Heart Fail. 2009; 11(2): 130-139, doi: 10.1093/eurjhf/hfn013, indexed in Pubmed: 19168510.

16. Tung RH, Camargo CA, Krauser $D$, et al. Amino-terminal pro-brain natriuretic peptide for the diagnosis of acute heart failure in patients with previous obstructive airway disease. Ann Emerg Med. 2006; 48(1): 66-74, doi: 10.1016/j.annemergmed.2005.12.022, indexed in Pubmed: 16781921.

17. Kelly AM, Holdgate A, Keijzers $G$, et al. Epidemiology, treatment, disposition and outcome of patients with acute exacerbation of COPD presenting to emergency departments in Australia and South East Asia: An AANZDEM study. Respirology. 2018; 23(7): 681-686, doi: 10.1111/resp.13259, indexed in Pubmed: 29394524.

18. Laribi S, Keijzers $G$, van Meer 0 , et al. AANZDEM and EURODEM study groups. Epidemiology of patients presenting with dyspnea to emergency departments in Europe and the Asia-Pacific region. Eur J Emerg Med. 2019; 26(5): 345-349, doi: 10.1097/MEJ.00000000000000571, indexed in Pubmed: 30169464.

19. Gori CS, Magrini L, Travaglino F, et al. Role of biomarkers in patients with dyspnea. Eur Rev Med Pharmacol Sci. 2011; 15(2): 229-240, indexed in Pubmed: 21434492.

20. Stoica $A$, Şorodoc V, Lionte $C$, et al. Acute cardiac dyspnea in the emergency department: diagnostic value of $\mathrm{N}$-terminal prohormone of brain natriuretic peptide and galectin-3. J Int Med Res. 2019; 47(1): 159-172, doi: 10.1177/0300060518798257, indexed in Pubmed: 30222016.

21. Morrison LK, Harrison A, Krishnaswamy $P$, et al. Utility of a rapid $B$-natriuretic peptide assay in differentiating congestive heart failure from lung disease in patients presenting with dyspnea. J Am Coll Cardiol. 2002; 39(2): 202-209, doi: 10.1016/s0735-1097(01)017442, indexed in Pubmed: 11788208.

22. Taylan M, Demir M, Kaya H, et al. Alterations of the neutrophil-lymphocyte ratio during the period of stable and acute exacerbation of chronic obstructive pulmonary disease patients. Clin Respir J. 2017; 11(3): 311-317, doi: 10.1111/crj.12336, indexed in Pubmed: 26096858. 